\title{
Designs for miniaturization of bending actuator utilizing hydrogen storage alloy
}

\author{
A. Kagawa*, K. Taniguchi, M. Yamamoto \\ Division of Chemistry and Materials Science, Nagasaki University, 1-14 Bunkyo-machi, Nagasaki, \\ 852-8521, Japan. \\ * Corresponding author. Tel.\&Fax: +81 095819 2640. E-mail address:akagawa@nagasaki-u.ac.jp (A. Kagawa).
}

\begin{abstract}
Bending behavior of a miniature actuator utilizing Pd-Ni hydrogen storage alloy (HSA) has been investigated on hydriding and dehydriding process. The actuator has a bimorph structure consisting of the HSA thin sheet and copper plating on one side of the sheet. Palladium sputtering on the back surface of the HSA significantly improves bending characteristics due to the catalytic action of palladium. The maximum bending velocity is obtained in the HSA with $10 \mu \mathrm{m}$ thick Cu-plating. The bending actuators having the same aspect ratio (length to width) and different dimensions show the same bending velocity and the critical aspect ratio of the actuator free from the constraint by deformation in width direction is around five. The bending behavior can be controlled by adjusting the surrounding hydrogen pressure.
\end{abstract}

Keywords: Actuator, Hydrogen Storage Alloy, Bending, Palladium, Nickel

\section{Introduction}

In recent years, minimally invasive treatments have been demanded for the improvement of QOL (quality of life) of patients in the medical field. In order to realize the invasive treatments, a small-size operation tool and its driving system using a miniature actuator will be required. Actuator utilizing hydrogen storage alloy (HSA) is a candidate of such an actuator.

HSAs exhibit a great volume expansion of 10 to $30 \%$ on hydrogenation, which is several tens times greater than thermal expansion, and are expected to be applied to a power actuator due to a large driving force caused by phase transformation, i.e., metal to metal hydride and vice versa.

On the structure of actuator utilizing HSA, Honjo et al. [1,2] have reported the bending behavior of the actuator utilizing the volume expansion of $\mathrm{LaNi}_{5}$ HSA. However, the bending velocity was not sufficient because a fine $\mathrm{LaNi}_{5}$ powder was embedded in a soft polymer matrix and the volume expansion of $\mathrm{LaNi}_{5} \mathrm{HSA}$ would not be utilized efficiently. $\mathrm{LaNi}_{5}$ and other well-known hydrogen storage materials, FeTi and magnesium base alloys, are intermetallic compounds and thus have a brittle nature that restricts the sample shape to powder form [3]. On the other hand, palladium (Pd) base solid solution HSAs have superior properties, such as excellent workability and high pulverization resistance, similar to vanadium base solid solution alloys [4], and a ribbon form is easily obtained. Therefore, an actuator which has a bimorph structure consisting of HSA layer and non-HSA layer, such as copper $(\mathrm{Cu})$-plating shown in Fig.1, is an alternative candidate of the actuator utilizing $\mathrm{LaNi}_{5}$ powder. Furthermore, the actuator utilizing Pd-base HSA has a feasibility of downsizing for application to small-size operation tools. Among the Pd-base alloys, Pd-Ni system has all-proportion miscibility and the absorption-desorption plateau pressures can be easily controlled by adjusting nickel content.

In the present work, the influence of Pd- sputtering onto the HSA surface of the actuator utilizing Pd-Ni HSA, thickness of Cu-plating and aspect ratio (length to width) of the actuator on the bending characteristics has been investigated.

\section{Experimental procedures}

Pd-13at\%Ni alloy having absorption/desorption plateau pressures around atmospheric pressure [5] was prepared by arc melting using a palladium plate (99.9 mass\%) and a nickel plate (99.9 mass\%) under an argon gas atmosphere. The alloy was remelted several times and was annealed at $1073 \mathrm{~K}$ for $15 \mathrm{~h}$ in vacuum for homogenization. A ribbon sample, $3 \mathrm{~mm}$ wide, $40 \mathrm{~mm}$ long and $20 \mu \mathrm{m}$ thick, was obtained by repeated rolling of the as-cast button alloy. In our preliminary experiment, it was found that a ribbon sample having the thickness greater than $40 \mu \mathrm{m}$ showed a slow bending motion due to the effect of Up-hill diffusion [6]. Furthermore, our preliminary experiment indicated that diffusion-bonding between HSA and non-HSA was 
harmful to obtain a fast bending motion of the actuator having a bimorph structure because of the formation of an interdiffusion layer between them or a reaction layer at their interface. Therefore, the thickness of $20 \mu \mathrm{m}$ was adopted and the diffusion-bonding heat treatment was eliminated in the present work. Bending actuator having a bimorph structure shown in Fig.1 was prepared by electroplating pure copper on one side of the ribbon sample. The shape change behavior was observed on hydriding under hydrogen gas pressure of $0.3 \mathrm{MPa}$ and on dehydriding in vacuum. The displacement of the top end of an actuator sample was measured on the movie images recorded in the video tape.

\section{Results and discussion}

\subsection{Effect of Pd-sputtering}

The bending behavior of actuator samples ( $20 \mu \mathrm{m}$-thick HSA ribbon and $40 \mu \mathrm{m}$-thick Cu-plating) without and with Pd-sputtering on the surface of the HSA was observed on hydriding and dehydriding process. Fig. 2 shows their bending motions on absorption of hydrogen. Pd-sputtering significantly enhances the bending velocity. Fig. 3 shows the time dependence of the displacement at the top-end of the actuators with and without Pd-sputtering. Bending velocity of the actuator sample with Pd-sputtering is about 12 times faster on absorption and 28 times faster on desorption than that of the actuator sample without Pd-sputtering. This difference is possibly caused by the catalytic action of Pd.

\subsection{Effect of Cu-plating thickness}

In order to elucidate the optimal thickness of Cu-plating, actuator samples having different Cu-plating thickness of 10 to $80 \mu \mathrm{m}$ were prepared. Fig. 4 shows the variation of the displacement at the top-end of the actuator samples having different Cu-plating thickness with time. The bending velocity, an average slope of the curve in the range of the displacement up to 8mm in Fig.4, is plotted in Fig.5.

With increasing Cu-plating thickness, the bending velocity is reduced on absorption. This is attributable to an increase in the rigidity of Cu-plating with increasing the Cu-plating thickness. On desorption, the bending velocity is expected to be raised by an elastic recovery force of the Cu-plating. However, as seen in Fig.5, the recovery motion is relatively small. When hydrogen atoms are discharged from the HSA surface, the lattice contraction occurs near the surface due to the escape of dissolved hydrogen and the shape change from a curved surface to a straight one on desorption process. This lattice shrinkage near the surface may hinder a further hydrogen diffusion from inside to the surface, resulting in a suppression of recovery motion. From the results in Fig.5, the thinner the Cu-plating, the higher the bending velocity on absorption although the deformation may not occur without Cu-plating. Therefore, the optimal thickness of Cu-plating is around $10 \mu \mathrm{m}$ where the maximum bending velocity is obtained.

\subsection{Effect of aspect ratio of the actuator samples}

In consideration of downsizing of the actuator, the effect of the aspect ratio (L/W : length (L) to width (W)) of ribbon samples on bending behavior was investigated.

When the aspect ratio is small, bending velocity is possibly reduced since the bending motion in width direction disturbs the bending motion in length direction as shown in Fig.6. On the other hand, reducing the width of actuator sample, i.e., increasing the aspect ratio leads to a lowering in the rigidity of the actuator. In order to elucidate the optimal aspect ratio free from the constraint by deformation in width direction, bending motion of actuator samples having a constant width $3 \mathrm{~mm}$ and different length was observed, where the sample $3 \mathrm{~mm}$ wide and $40 \mathrm{~mm}$ long $(\mathrm{L} / \mathrm{W}>10)$ is taken as a standard sample. The samples were subjected to Pd-sputtering treatment and Cu-plating thickness was $40 \mu \mathrm{m}$. Figure 7a shows an appearance of bending motion and arrows show the movement at the top-end and the locations $10 \sim 30 \mathrm{~mm}$ from the bottom of the standard sample. Fig.7b shows the time dependence of the displacement. In Fig.7b, solid curves show the displacement at the top-end of the samples 10,15, 20, 30 and $40 \mathrm{~mm}$ long. In the figure, displacement at the locations $10 \sim 30 \mathrm{~mm}$ in the 40 mm-long standard sample (Fig.7a) is also given by broken curves for comparison. The solid curves coincide well with broken curves for the samples longer than $15 \mathrm{~mm}(\mathrm{~L} / \mathrm{W}=5)$, while the $10 \mathrm{~mm}$-long sample exhibits a displacement smaller than that at the location of $10 \mathrm{~mm}$ in the $40 \mathrm{~mm}$-long sample shown by broken curve. This may be caused by the constraint from the motion in width direction. From the results shown in Fig.7, it is known that the critical L/W of the actuator which is free from the constraint by deformation in width direction is around five. Displacement at the same location of actuator samples with different dimensions and the same aspect ratio 
is shown in Fig.8. It is seen that the bending velocity is approximately equal for the samples having the same aspect ratio but different dimensions.

\subsection{Control of bending behavior of the actuator}

Since the amount of hydrogen absorbed in HSA varies with the hydrogen pressure of surroundings, the bending behavior of the actuator can be controlled by adjusting the hydrogen pressure. In order to develop the motion control technique for the actuator utilizing HSA, the bending behavior of the actuator ( $2 \mathrm{~mm} \mathrm{x} 10 \mathrm{~mm} \mathrm{x}$ $40 \mu \mathrm{m})$ at different hydrogen pressure was examined. Fig.9 shows the time dependence of the displacement at the top-end of the actuator on absorption when hydrogen pressure was introduced stepwise at every $0.05 \mathrm{MPa}$ in the range from 0.05 to $0.3 \mathrm{MPa}$. The bending velocity decreases gradually with increasing the hydrogen pressure. This is considered to be caused by well-known "Gorsky effect" [7], i.e., when a sample absorbs hydrogen, a strain gradient is generated across the thickness and dissolved hydrogen atoms diffuse toward the surface with a convex shape, through which hydrogen atoms are absorbed. From the results given in Fig.9, it is indicated that the bending behavior of the actuator can be controlled by adjusting the hydrogen pressure.

\section{Conclusions}

The bending actuator utilizing volume changes of Pd-Ni HSA on hydrogen absorption and desorption has been developed. In view of the application of the actuator to small-size operation tools in the medical field, the influence of Pd-sputtering, thickness of Cu-plating and aspect ratio (length to width) of the actuator on the bending characteristics has been investigated.

The actuator which has a bimorph structure consisting of the HSA thin sheet and copper plating on one side of the sheet shows a reversible bending motion. Pd-sputtering on the HSA surface remarkably improves the bending characteristics due to the catalytic effect of Pd. As for the effect of the thickness of Cu-plating, bending velocity decreases with increasing the plating thickness on absorption and the maximum bending velocity is obtained in the HSA with $10 \mu \mathrm{m}$ thick Cu-plating. On desorption, recovery motion is relatively small due to a lattice contraction near the HSA surface. The bending actuators having the same aspect ratio (length/width) and different dimensions show the same bending velocity at the same location. The critical aspect ratio of the actuator free from the constraint by deformation in width direction is around five. Since the amount of hydrogen absorbed in HSA varies with surrounding hydrogen pressure, the bending behavior of the actuator can be controlled by adjusting the hydrogen pressure.

\section{Acknowledgement}

This research was partially supported by the Ministry of Education, Culture, Sports, Science and Technology, Grant-in-Aid for Scientific Research (C), 24500512, 2012.

\section{References}

[1] T. Honjo, H. Yabe, S. Tsubuteishi, H. Uchida, Y. Nishi, J. Jpn. Inst. Met. 67 (4) ( 2003) 145.

[2] T. Honjo, H. Uchida, Y. Matsumura, Y. Nishi, J. Jpn. Inst. Met. 68 (4) (2004) 58.

[3] S. L. Li, P. Wang, W. Chen, G. Luo, D. M. Chen, K. Yang, J. Alloys Compd. 485 (2009) 867.

[4] A. Kagawa, E. Ono, T. Kusakabe and Y. Sakamoto: J. Less-Common Met. 172-174(1991) 64.

[5] M. Mizumoto, T. Ohgai, A. Kagawa, J. Alloys Compd. 482 (2009) 416.

[6] B. Baranowski, J. Less-Common Met. 154 (1989) 329.

[7] K.Kandasamy, F.A.Lewis, Int. J. Hydrogen Energy, 24 (1999) 763. 


\section{Hydrogen Storage Alloy}

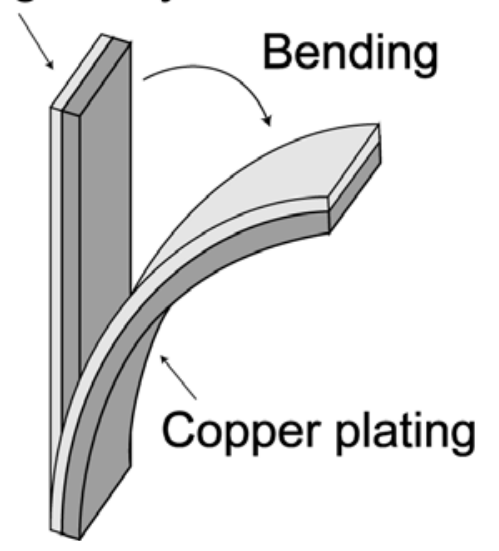

Fig.1. Schematic of a bending actuator utilizing hydrogen storage alloy. 


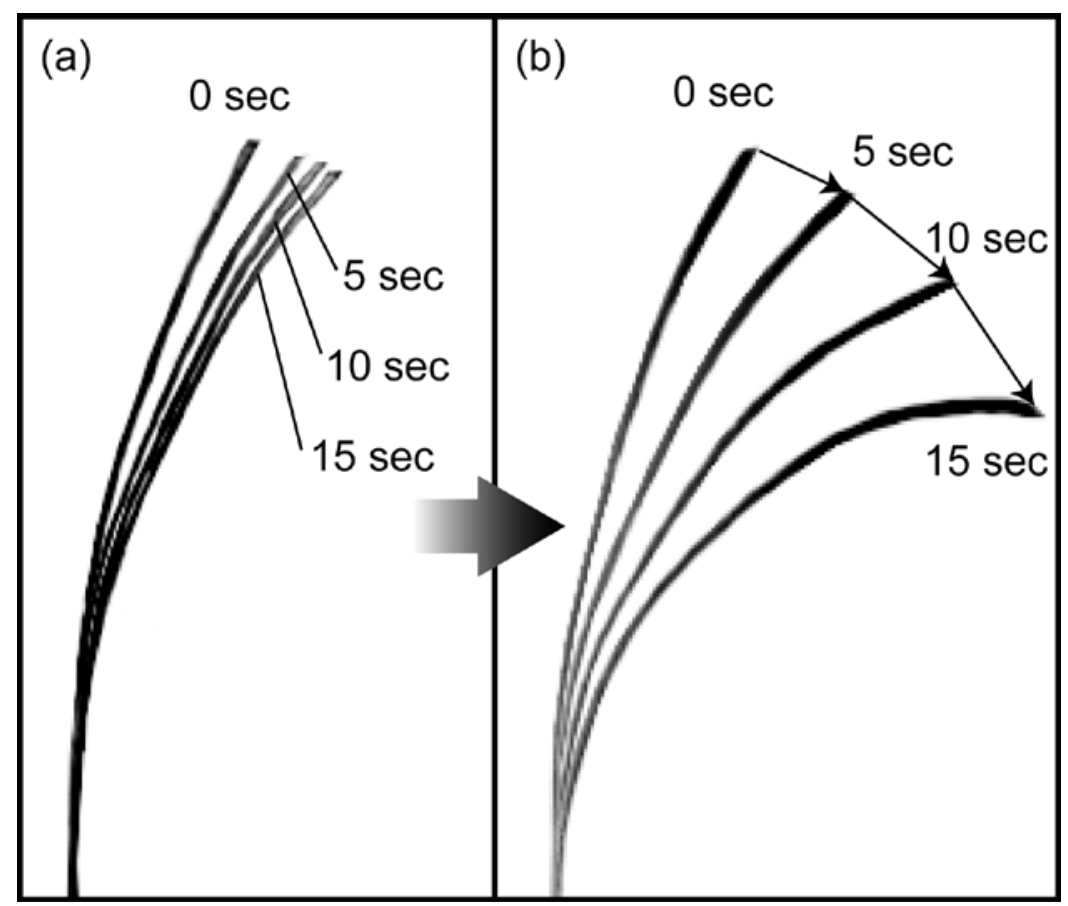

Fig.2. Bending motion of the Pd-Ni HSA actuator samples on hydrogen absorption. a) without and $b$ ) with $\mathrm{Pd}$-sputtering. 


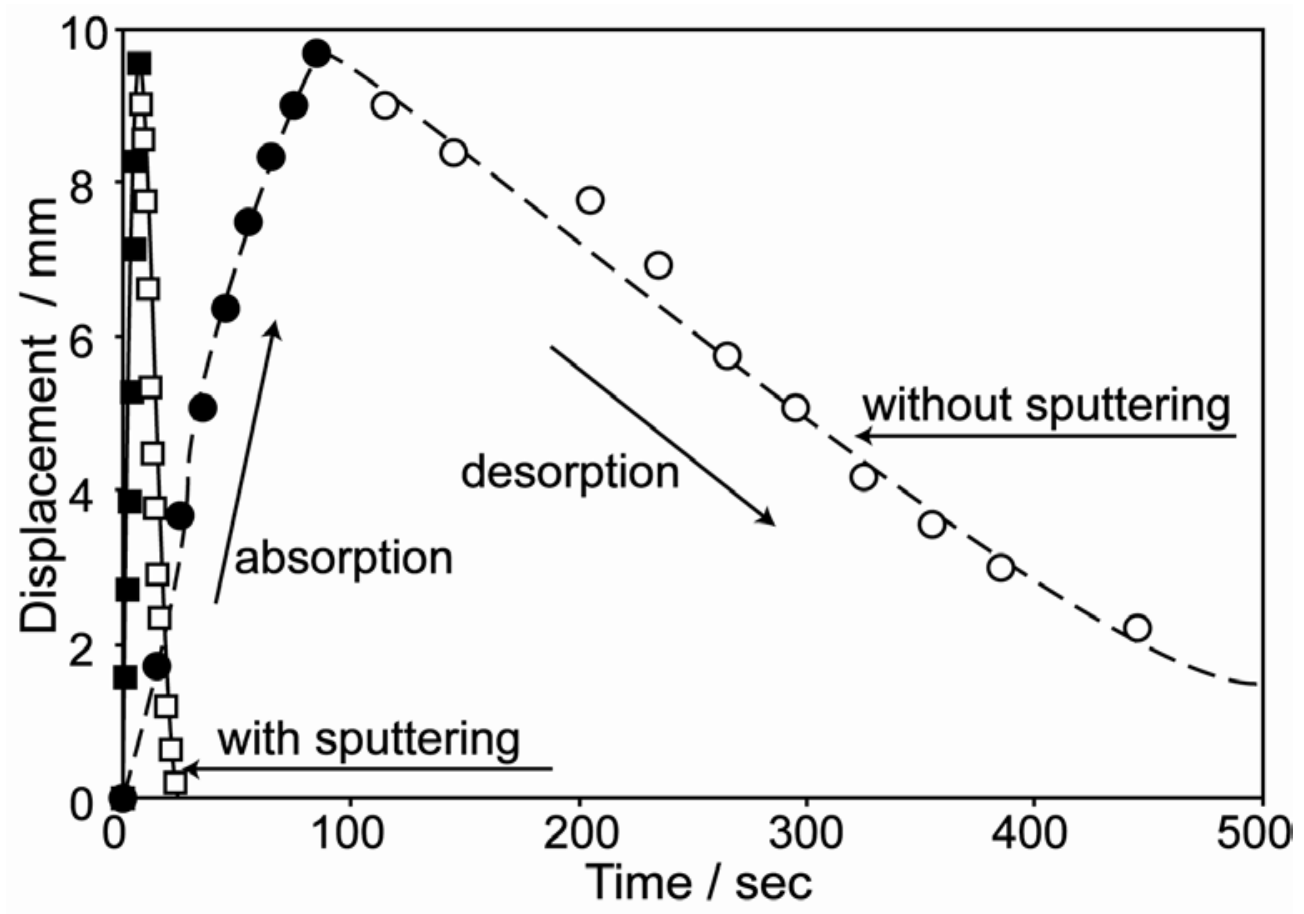

Fig.3. Displacement of the top-end of the Pd-Ni HSA actuator samples with and without Pd-sputtering. 


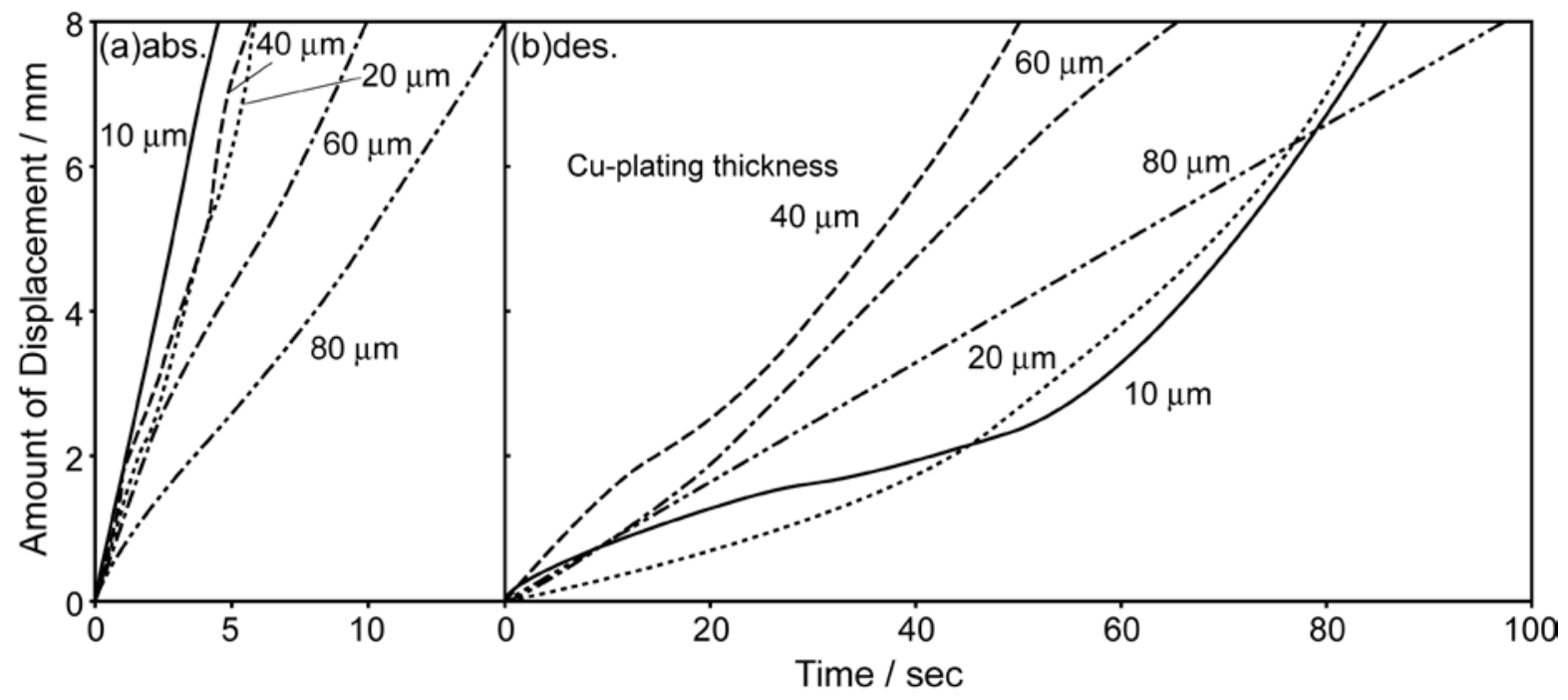

Fig.4. Effect of the thickness of Cu-plating on the displacement of the top-end of the Pd-Ni HSA actuator samples. 


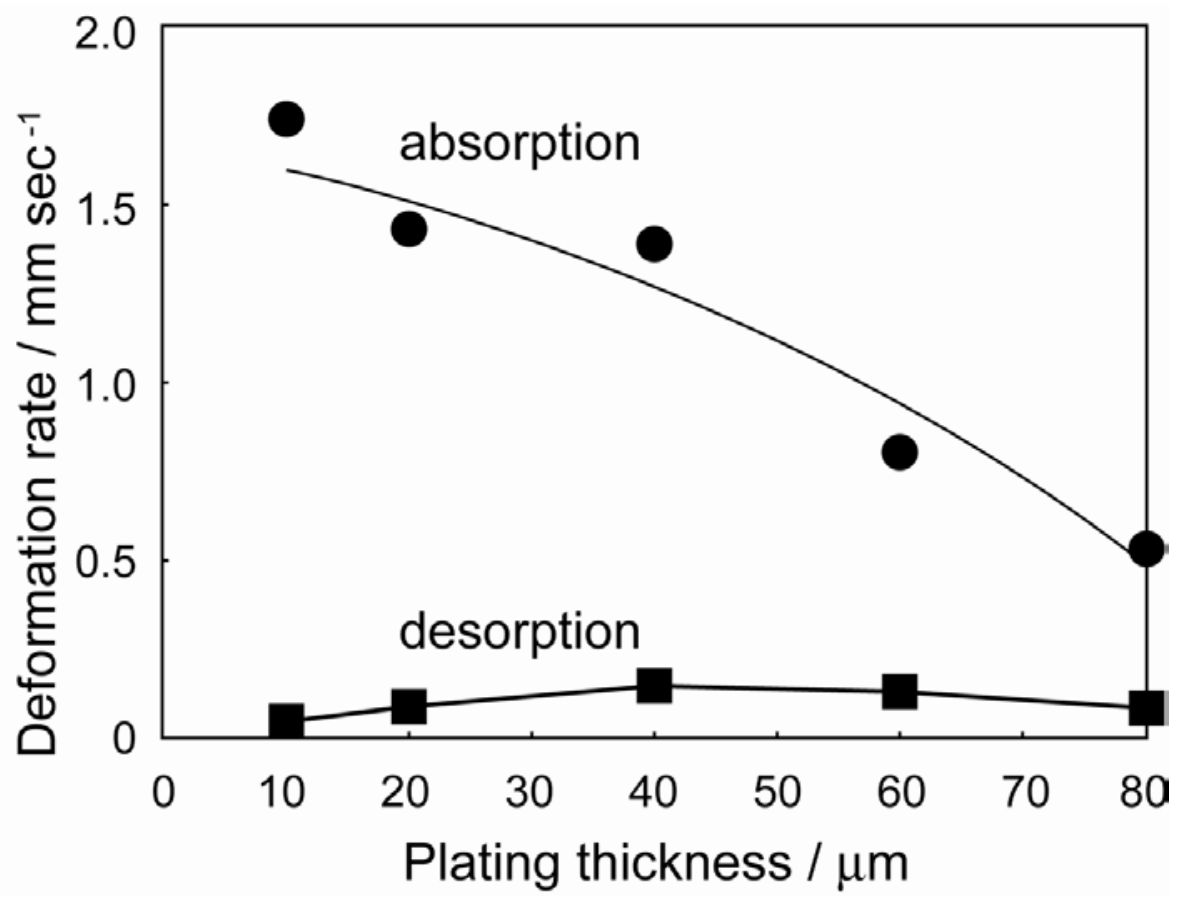

Fig.5. Dependence of bending and recovery velocities of the Pd-Ni HSA actuator samples on Cu-plating thickness. 


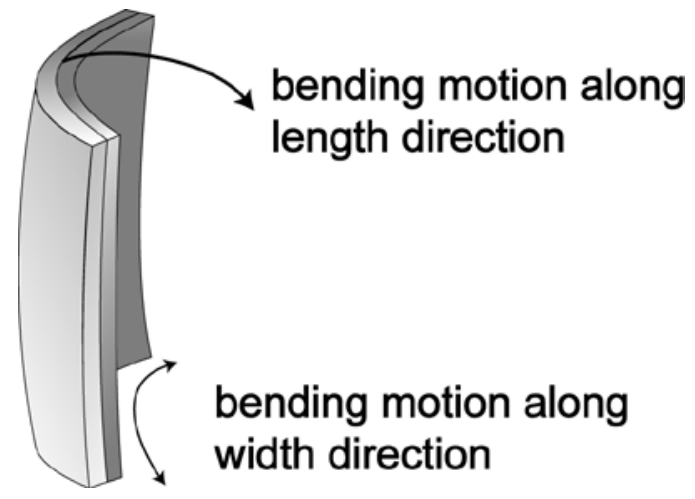

Fig.6. Schematic illustration of superimposed bending motions along length and width directions. 
a)
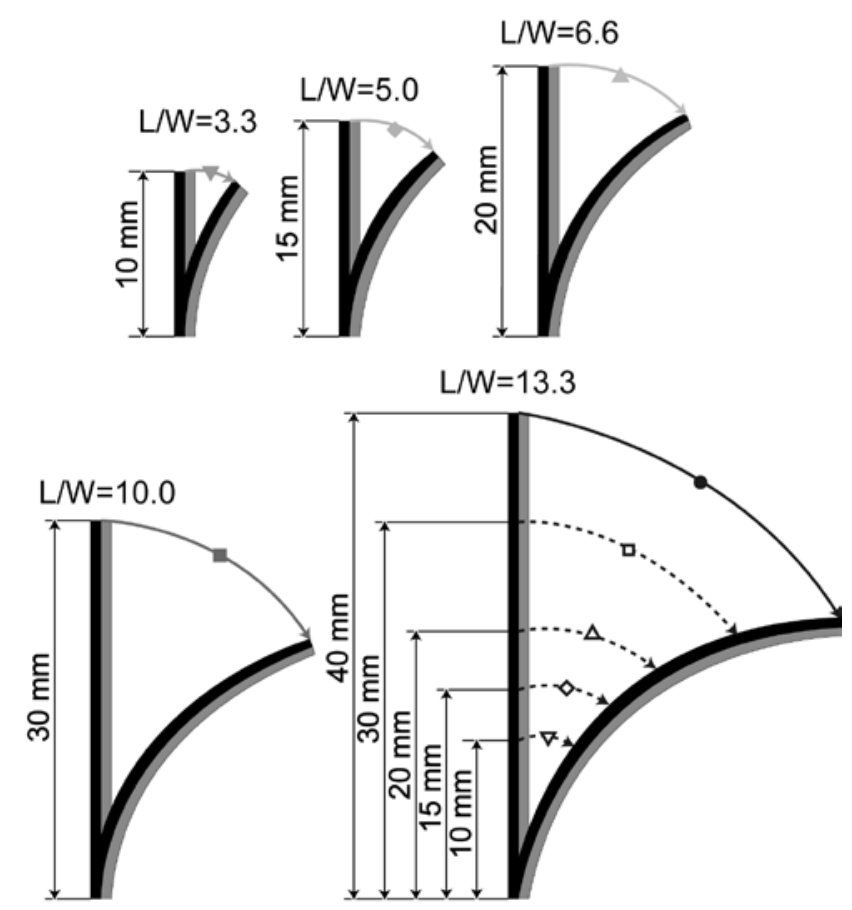

b)

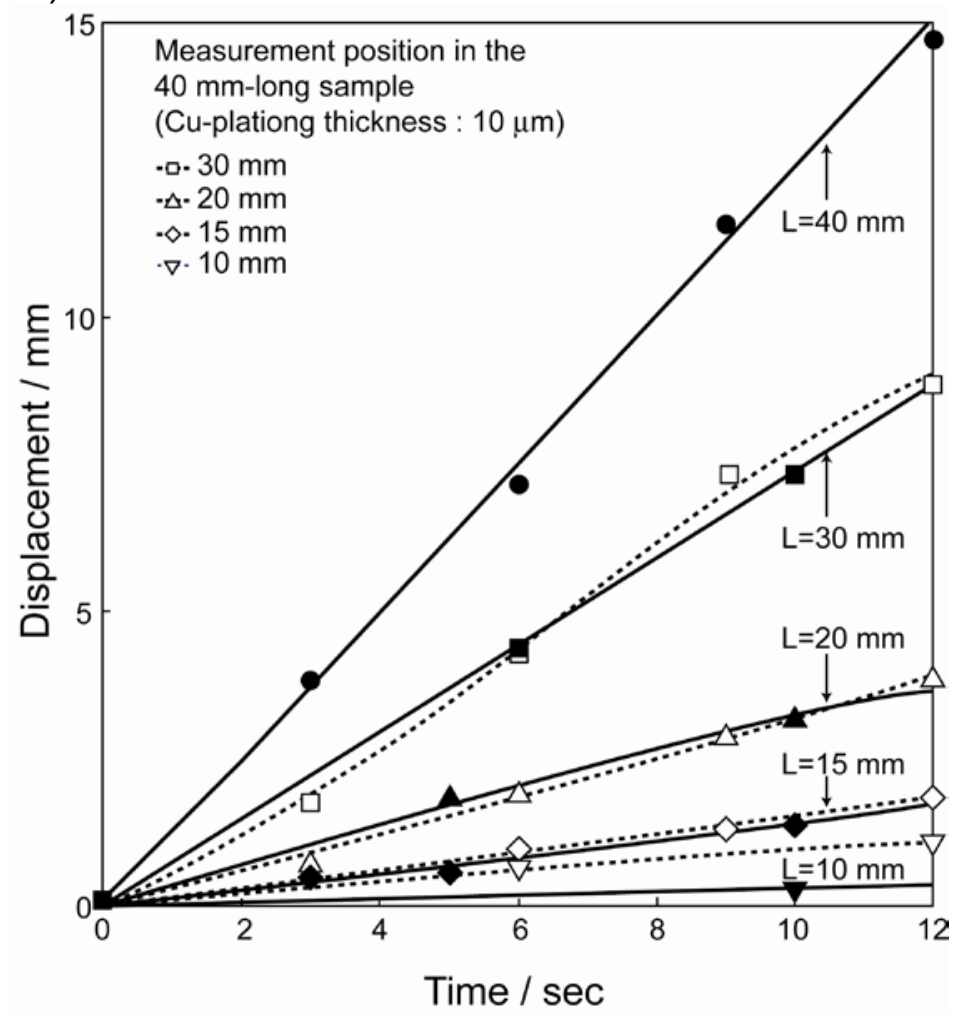

Fig.7. Change in the displacement at the top-end and different locations of the $\mathrm{Pd}-\mathrm{Ni}$ HSA actuator samples having different aspect ratios. (on absorption) 
a)

b)
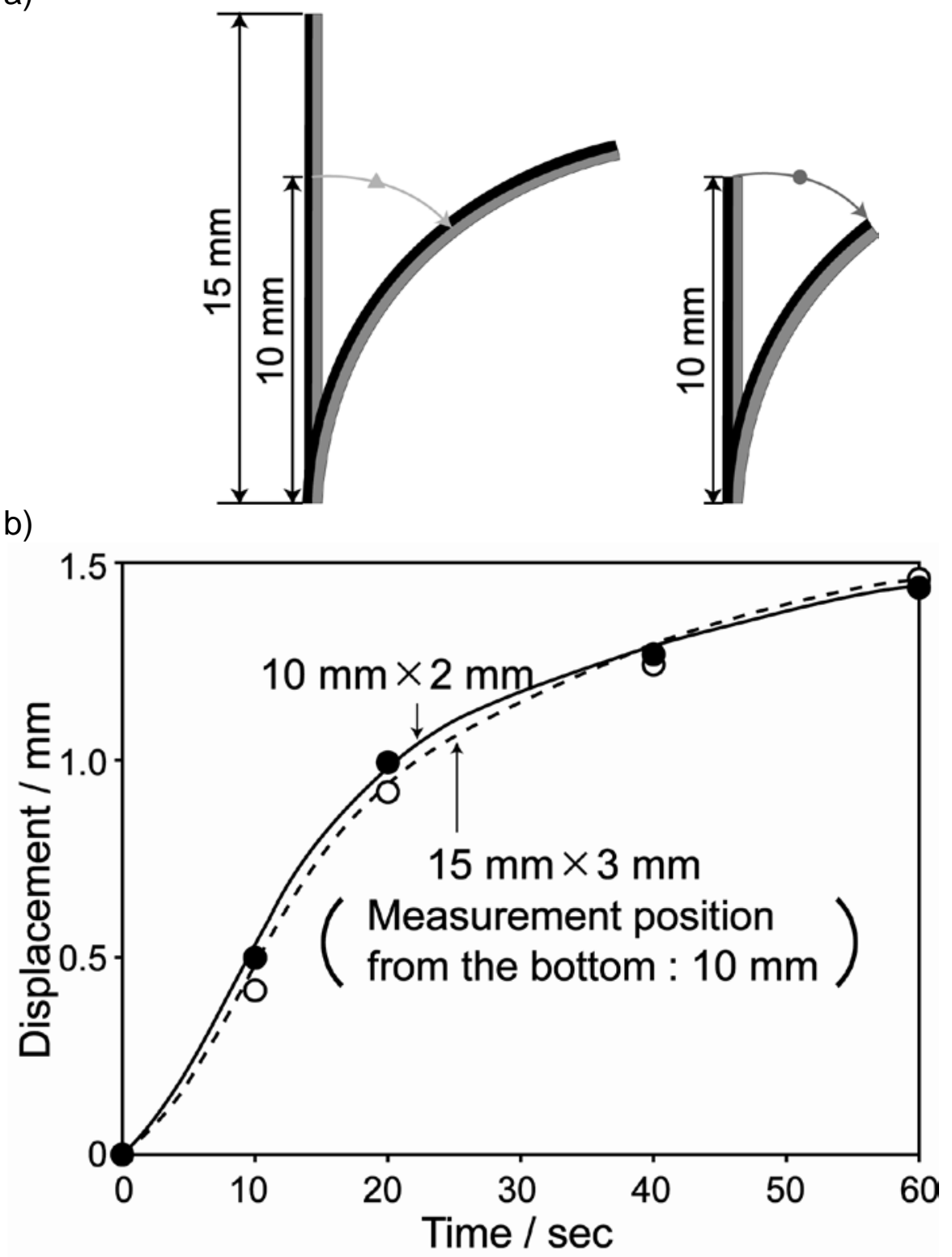

Fig.8. Displacement at the same location of the Pd-Ni HSA actuator samples with different dimensions and the same aspect ratio. 


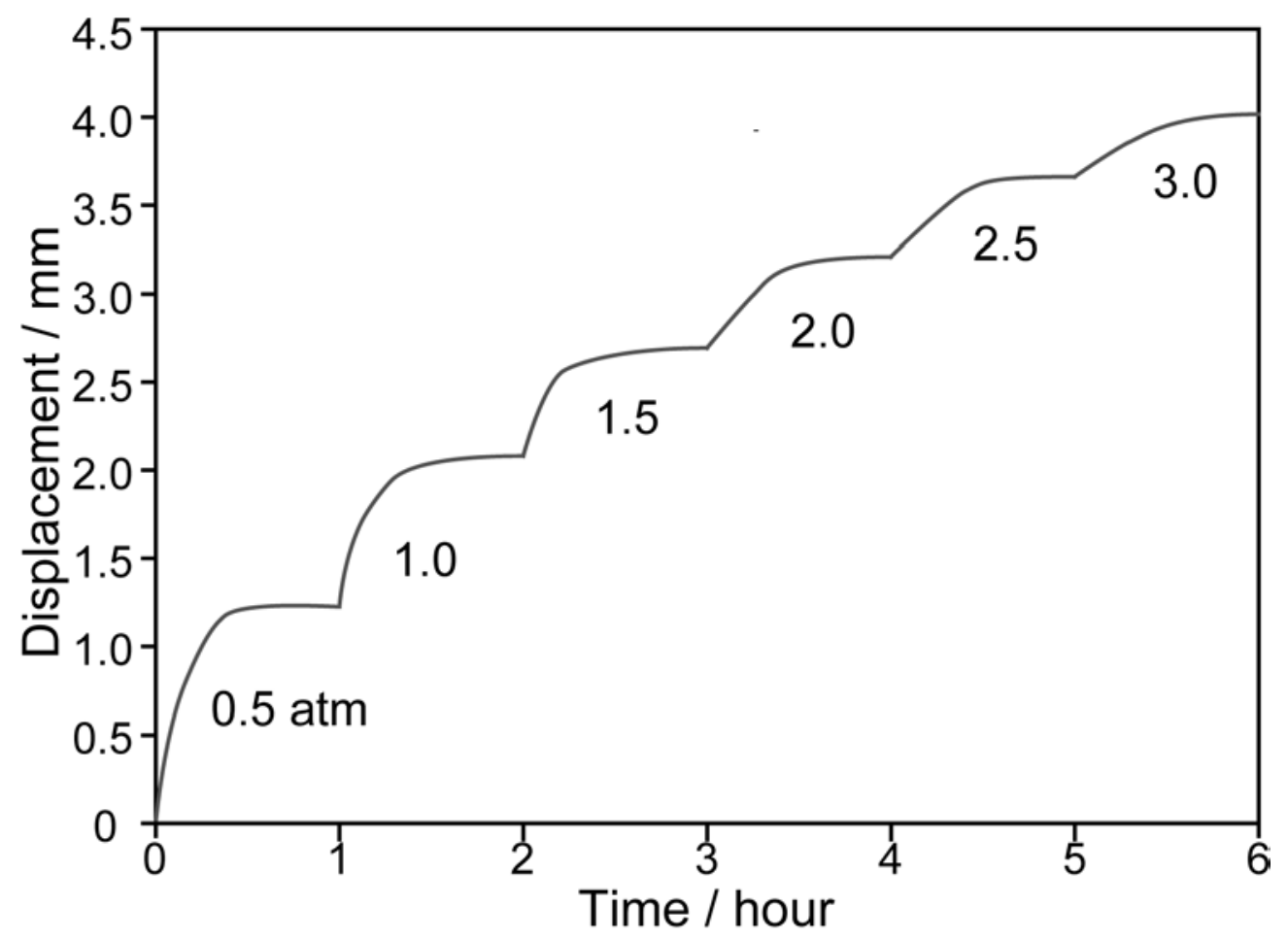

Fig.9. Change in the displacement of the top-end of the Pd-Ni HSA actuator sample with time on absorption. 


\section{Captions for illustrations}

Fig.1. Schematic of a bending actuator utilizing hydrogen storage alloy.

Fig.2. Bending motion of the Pd-Ni HSA actuator samples on hydrogen absorption. a) without and b) with Pd-sputtering.

Fig.3. Displacement of the top-end of the Pd-Ni HSA actuator samples with and without Pd-sputtering.

Fig.4. Effect of the thickness of Cu-plating on the displacement of the top-end of the Pd-Ni HSA actuator samples.

Fig.5. Dependence of bending and recovery velocities of the Pd-Ni HSA actuator samples on Cu-plating thickness.

Fig.6. Schematic illustration of superimposed bending motions along length and width directions.

Fig.7. Change in the displacement at the top-end and different locations of the Pd-Ni HSA actuator samples having different aspect ratios. (on absorption)

Fig.8. Displacement at the same location of the Pd-Ni HSA actuator samples with different dimensions and the same aspect ratio.

Fig.9. Change in the displacement of the top-end of the Pd-Ni HSA actuator sample with time on absorption. 